\title{
Understanding the fusion cross section among light nuclei around the Coulomb barrier
}

\author{
Antonio Del Zoppo ${ }^{1, \star}$ and Marco La Cognata ${ }^{1, \star \star}$ \\ ${ }^{1}$ Istituto Nazionale di Fisica Nucleare - Laboratori Nazionali del Sud, via S. Sofia 62, 95123 Catania, Italy
}

\begin{abstract}
In this work we investigate fusion induced by a radioactive ${ }^{8} \mathrm{Li}$ projectile on a ${ }^{4} \mathrm{He}$ gas target, at center-of-mass energies between 0.6 and $5 \mathrm{MeV}$. The main result is the tendency of the dimensionless fusion cross section $\frac{\sigma}{\pi \lambda^{2}}$ to form well visible plateaus alternated to steep rises. This is likely to be the most genuine consequence of the discrete nature of the intervening angular momenta observed so far in fusion reactions right above the Coulomb barrier. A partial-wave analysis, exclusively based on a pure quantal penetration fusion model, identifies a remarkably low-height barrier. Indeed, these plateaus allow enhanced experimental sensitivity to the fusion barrier given that the most barrier-sensitive lowest partial waves are well separated. We expect that the present results for ${ }^{8} \mathrm{Li}+{ }^{4} \mathrm{He}$ will promote further investigations of the fusion reaction mechanism between very light ions at energies much below the interaction barrier. For the moment, we believe that understanding the plateau origin in the cross section above the barrier will almost certainly be useful to corroborate the extrapolation to the important astrophysical region below the Coulomb barrier, not only in the case of the ${ }^{8} \mathrm{Li}+{ }^{4} \mathrm{He}$ fusion but also for other systems, such as the ${ }^{12} \mathrm{C}+{ }^{12} \mathrm{C}$.
\end{abstract}

\section{Introduction}

Fusion between light ions plays an important role in many astrophysical sites such as, for instance, quiescent-burning stars, including massive stars beyond H-burning, type Ia supernovae and surface explosions of neutron stars. Exotic scenarios like pycnonuclear reactions occurring in high density matter, when nuclei are frozen in lattice structures, are also dominated by ${ }^{12} \mathrm{C}$ and ${ }^{16} \mathrm{O}$ induced fusion processes [1]. A exhaustive review can be find, for instance, in Ref.[2]. Fusion between light nuclei is also of growing importance in the field of energy production by means, among others, of inertial confinement [3]. However, energies of astrophysical interest reach at most few MeV even in the case of explosive scenarios, when temperatures as large as $10^{9} \mathrm{~K}$ are achieved [2], which are usually lower or comparable with the corresponding Coulomb barriers. Therefore, extrapolation sometimes supported by nuclear reaction theory is often necessary to establish the lowenergy trend of the cross section at astrophysical energies. This can introduce large systematic errors if the underpinning theory turns out to be an inaccurate description of the physical processes, possibly causing large deviations of the theoretical models using the extrapolated cross sections from observations. Therefore, the problem of extrapolation has to be challenged to provide as much accurate as possible cross section previsions for astrophysics and applied physics. In this work we investigate the low energy trend of the ${ }^{8} \mathrm{Li}+\alpha$ fusion reaction. The need of a

\footnotetext{
^e-mail: delzoppo@lns.infn.it

$\star \star$ e-mail: lacognata@lns.infn.it
}

realistic extrapolation of the cross section to energies below about $1 \mathrm{MeV}$ and the existence of some incompatible data sets have triggered a close examination of the origin of the oscillations characterising its cross section. A detailed discussion on the ${ }^{8} \mathrm{Li}+\alpha$ cross section measurement and on the discrepancy between the different data sets can be found in Refs.[4-10].

\section{The ${ }^{8} \mathrm{Li}+\alpha$ fusion cross section}

The ${ }^{8} \mathrm{Li}+\alpha$ fusion has important astrophysical applications in explosive scenarios such as the big bang nucleosynthesis and the heavy-element production through the r-process. If neutron-rich regions formed before the onset of nucleosynthesis (inhomogeneous model, see, for instance, Refs.[11, 12]), the ${ }^{8} \mathrm{Li}+\alpha$ capture might have acted as a bridge towards the production of elements heavier than lithium, otherwise synthesised in negligible amount in standard big band scenarios. Similarly, in neutron rich environments where r-process can take place [13, 14], seed nuclei eventually burnt to heavier elements in the fast neutron captures could be obtained through $\alpha$-capture reactions, in particular on ${ }^{8} \mathrm{Li}$.

Fig.1a) displays the ${ }^{8} \mathrm{Li}+\alpha$ concordant data set as established in [7]. Such fusion cross section clearly shows a oscillating pattern, with a period of some $\mathrm{MeV}$, recalling the ${ }^{12} \mathrm{C}+{ }^{12} \mathrm{C}$ data [17], the literature case where such oscillations are most evident, given in Fig.1b). In Figs.1c) and d) exhibit the same data expressed in the form of dimensionless cross section in units of $\pi t^{2}$. Both figures indicate the existence of nearly horizontal plateaus clearly 


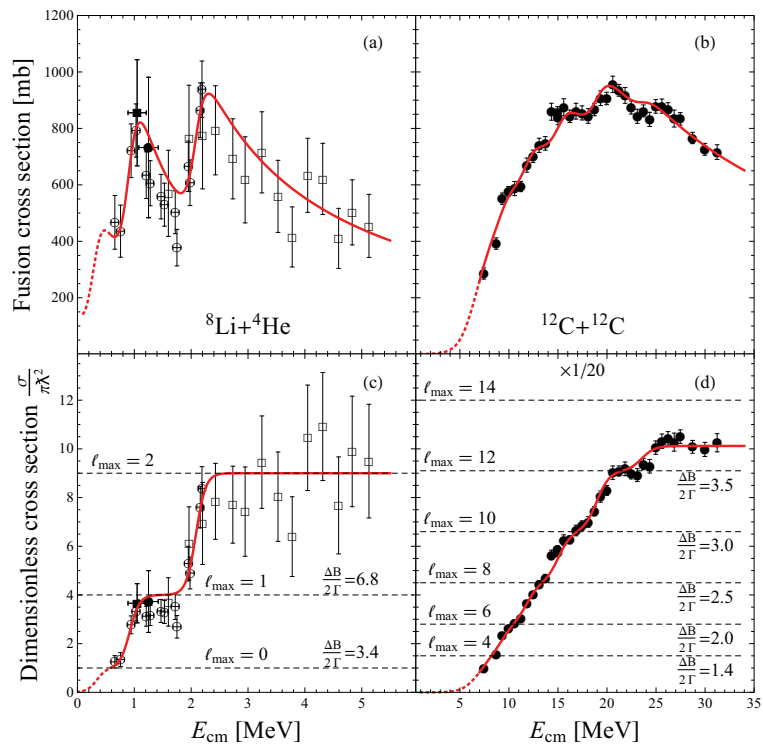

Figure 1. Panel a) ${ }^{8} \mathrm{Li}+\alpha$ fusion cross section. Experimental data are taken from: Ref.[15] open squares, Ref.[16] open circles, Ref.[4] filled circle, Ref.[6, 8] filled square. Panel b) ${ }^{12} \mathrm{C}+{ }^{12} \mathrm{C}$ fusion cross section. Experimental data are taken from Ref.[17]. Panel c) and panel d) dimensionless cross sections for the ${ }^{8} \mathrm{Li}+\alpha$ and ${ }^{12} \mathrm{C}+{ }^{12} \mathrm{C}$ channels, respectively. In panel d) all ordinates are divided by 20 to keep the same vertical axis. The solid curves are the results of the MINUIT data fits using Eq.1. Dashed curves are extrapolation to zero energy

alternate with steep rises that, in the case of ${ }^{12} \mathrm{C}+{ }^{12} \mathrm{C}$ data are apparent only above about $20 \mathrm{MeV}$.

As already done for the ${ }^{12} \mathrm{C}+{ }^{12} \mathrm{C}$ fusion cross section, we assume that the oscillatory structures arise from the progressive addition of higher partial waves with increasing energy, as already discussed in Refs.[18-20]. The detailed analysis is reported in Ref.[21]. To probe such interpretation for the ${ }^{8} \mathrm{Li}+\alpha$ data, we make use of a formalism that explicitly takes into account the angular momentum of the relative motion $l$ and introduce a sharp angular momentum cut-off that allows all of the flux crossing the interaction barrier to fuse for values of $l \leq l_{\max }$. Accordingly, the dimensionless cross section takes the form:

$$
\frac{\sigma_{f}}{\pi \hbar^{2}}=\Delta l \sum_{l=0, \Delta l}^{l_{\max }}(2 l+1) T_{l}
$$

where $\Delta l=1$ for ${ }^{8} \mathrm{Li}+\alpha$, whereas $\Delta l=2$ for identical even-even nuclei, for which all odd partial waves vanish. In Eq. $1, T_{l}$ is the energy-dependent penetration probability of the $l-t h$ partial wave through the interaction barrier. In particular, when all $T_{l}$ competing to a given energy tend to unity, plateaus may appear taking the following values:

$$
\frac{\sigma_{f}}{\pi \hbar^{2}} \rightarrow\left(l_{\max }+1\right)^{2}
$$

for $\Delta l=1$ and

$$
\frac{\sigma_{f}}{\pi \hbar^{2}} \rightarrow\left(l_{\max }+1\right)\left(l_{\max }+2\right)
$$

Table 1. Barrier height, intrinsic width and moment of inertia parameter sets determined for both reactions using MINUIT data fitting procedure.

\begin{tabular}{llll}
\hline Reaction & $B_{0}(\mathrm{MeV})$ & $\Gamma(\mathrm{MeV})$ & $I\left(\mathrm{MeV}^{-1}\right)$ \\
\hline${ }^{8} \mathrm{Li}+\alpha$ & $0.34 \pm 0.05$ & $0.08 \pm 0.02$ & $1.72 \pm 0.07$ \\
${ }^{12} \mathrm{C}+{ }^{12} \mathrm{C}$ & $5.70 \pm 0.12$ & $0.65 \pm 0.14$ & $5.88 \pm 0.10$ \\
\hline
\end{tabular}

for $\Delta l=2$. The plateau values calculated with Eq. 2 and Eq. 3 for the ${ }^{8} \mathrm{Li}+\alpha$ and ${ }^{12} \mathrm{C}+{ }^{12} \mathrm{C}$ systems, respectively, are given in Fig.1c) and d) for relevant values of $l_{\text {max }}$ and marked with short-dashed lines.

Very good agreement is found in the ${ }^{8} \mathrm{Li}+\alpha$ dimensionless cross section, while only for $l_{\max }=10$ and 12 there is significant evidence of the occurrence of the plateaus for the ${ }^{12} \mathrm{C}+{ }^{12} \mathrm{C}$ system. This is a clear sign that the oscillatory structures plausibly arise from the progressive addition of higher partial waves with increasing energy, rather than from properties of the compound nuclear system in the ${ }^{8} \mathrm{Li}+\alpha$ case under examination. Indeed, the results for both processes are absolutely compatible if the $\Delta B / 2 \Gamma$ parameter is considered for both cases, where $\Delta B$ is the difference of barrier heights for two subsequents allowed values of the orbital angular momenta and $\Gamma$ is the barrier width assumed constant [21]. To obtain them, we fitted the dimensionless cross sections using Eq.1, taking

$$
T_{l}\left(E_{c m}\right)=\left[1+\exp \left(\frac{B_{l}-E_{c m}}{\Gamma}\right)\right]^{-1},
$$

where $B_{l}=B_{0}+\frac{l(l+1)}{2 I}$. The fitting parameter are given in Tab.1. The resulting $\Delta B / 2 \Gamma$ parameters are shown in Fig.1c) and d); the comparison of the values for the ${ }^{8} \mathrm{Li}+$ $\alpha$ and ${ }^{12} \mathrm{C}+{ }^{12} \mathrm{C}$ systems demonstrates that plateaus are already visible in the former case for $l_{\max }=0$ since the indicator value $\Delta B / 2 \Gamma=3.4$, a number obtained only for $l_{\text {max }}=12$ for ${ }^{12} \mathrm{C}+{ }^{12} \mathrm{C}$ fusion.

\section{Concluding remarks}

In this work we obtain unprecedented experimental information on the ${ }^{8} \mathrm{Li}+\alpha$ fusion at center-of-mass energies $E_{c m}$ between 0.6 and $5 \mathrm{MeV}$, right above the Coulomb barrier. Experimental dimensionless cross section exhibits two well visible plateaus alternated to steep rises; a fusion model that solely includes the action of the relative motion angular momenta turns out to be a proper description of the reaction mechanism. Therefore, this appears as the most genuine consequence of the discrete nature of subsequent angular momenta observed so far in fusion reactions.

Concerning barrier shape assessment, for ${ }^{8} \mathrm{Li}+\alpha$ neither coupled-channel effects nor collision partner deformations should significantly contribute [22], while for ${ }^{12} \mathrm{C}+{ }^{12} \mathrm{C}$ coupled channels calculations were not able to reproduce fusion data [18]. Consequently, a pure single barrier penetration fusion model is adopted here for both reactions. Hill-Wheeler barrier penetration formula is used and most of the barrier parameters determined here for ${ }^{8} \mathrm{Li}+\alpha$ fusion are consistent with those of ${ }^{12} \mathrm{C}+{ }^{12} \mathrm{C}$, with the possible exception of $B_{0}$. This work identifies a 
notably low Coulomb barrier that can be possibly linked to the presence of the loosely bound ${ }^{8} \mathrm{Li}$.

\section{References}

[1] D.G. Yakovlev, L.R. Gasques, A.V. Afanasjev, M. Beard, M. Wiescher, Phys. Rev. C 74, 035803 (2006), astro-ph/0608488

[2] C. Iliadis, Nuclear Physics of Stars (Wiley-VCH Verlag, 2007)

[3] R. Betti, O.A. Hurricane, Nature Physics 12, 435 (2016)

[4] S. Cherubini, P. Figuera, A. Musumarra, C. Agodi, R. Alba, L. Calabretta, L. Cosentino, A. Del Zoppo, A. Di Pietro, M. La Cognata et al., European Physical Journal A 20, 355 (2004)

[5] A. Del Zoppo, P. Figuera, A. Musumarra, N. Colonna, R. Alba, C. Bonomo, S. Cherubini, L. Cosentino, A. Di Pietro, M. Gulino et al., Nuclear Instruments and Methods in Physics Research A 581, 783 (2007)

[6] M. La Cognata, A. Del Zoppo, P. Figuera, A. Musumarra, R. Alba, S. Cherubini, N. Colonna, L. Cosentino, V. Crucillà, A. di Pietro et al., Physics Letters B 664, 157 (2008)

[7] M. La Cognata, A. Del Zoppo, R. Alba, S. Cherubini, N. Colonna, A. Di Pietro, P. Figuera, M. Gulino, L. Lamia, A. Musumarra et al., Astrophys. J. Lett. 706, L251 (2009), 0911.2585

[8] M. La Cognata, A. Del Zoppo, R. Alba, S. Cherubini, N. Colonna, A. Di Pietro, P. Figuera, M. Gulino, L. Lamia, A. Musumarra et al., Journal of Physics G Nuclear Physics 37, 105105 (2010)
[9] M. La Cognata, A. Del Zoppo, Astrophys. J. 736, 148 (2011)

[10] M. La Cognata, A. Del Zoppo, Physica Scripta Volume T 150, 014019 (2012)

[11] R.A. Malaney, W.A. Fowler, Astrophys. J. 333, 14 (1988)

[12] J.F. Lara, T. Kajino, G.J. Mathews, Phys. Rev. D 73, 083501 (2006), astro-ph/0603817

[13] S.E. Woosley, R.D. Hoffman, Astrophys. J. 395, 202 (1992)

[14] T. Sasaqui, K. Otsuki, T. Kajino, G.J. Mathews, Astrophys. J. 645, 1345 (2006), astro-ph/0507393

[15] R.N. Boyd, I. Tanihata, N. Inabe, T. Kubo, T. Nakagawa, T. Suzuki, M. Yonokura, X.X. Bai, K. Kimura, S. Kubono, Physical Review Letters 68, 1283 (1992)

[16] X. Gu, R.N. Boyd, M.M. Farrell, J.D. Kalen, C.A. Mitchell, J.J. Kolata, M. Belbot, K. Lamkin, K. Ashktorab, F.D. Becchetti et al., Physics Letters B 343, 31 (1995)

[17] D.G. Kovar, D.F. Geesaman, T.H. Braid, Y. Eisen, W. Henning, T.R. Ophel, M. Paul, K.E. Rehm, S.J. Sanders, P. Sperr et al., Phys. Rev. C 20, 1305 (1979)

[18] H. Esbensen, Phys. Rev. C 85, 064611 (2012), 1206.1068

[19] C.Y. Wong, Phys. Rev. C 86, 064603 (2012)

[20] N. Rowley, K. Hagino, Phys. Rev. C 91, 044617 (2015)

[21] A. Del Zoppo, M. La Cognata, Physics Letters B 753, 449 (2016)

[22] K. Hagino, N. Takigawa, Progress of Theoretical Physics 128, 1061 (2012), 1209.6435 